\title{
Densities and Viscosities of Mixtures of Two Ionic Liquids Containing a Common Cation
}

\author{
Hugo F. D. Almeida ${ }^{1,2}$, José N. Canongia Lopes ${ }^{1,3}$, Luís P. N. Rebelo ${ }^{1}$, João A. P. \\ Coutinho $^{2}$, Mara G. Freire ${ }^{2}$ Isabel M. Marrucho ${ }^{1,3^{*}}$
}

\footnotetext{
${ }^{1}$ Instituto de Tecnologia Química e Biológica António Xavier, Universidade Nova de Lisboa, 2780-157 Oeiras, Portugal

${ }^{2} \mathrm{CICECO}$ - Instituto de Materiais de Aveiro, Departamento de Química, Universidade de Aveiro, 3810-193 Aveiro, Portugal

${ }^{3}$ Centro de Química Estrutural, Instituto Superior Técnico, Av. Rovisco Pais 1, 1049-

001 Lisboa, Portugal
}

${ }^{*}$ Corresponding author: Isabel M. Marrucho

Tel: +351-214469720

Fax: +351-214411277

E-mail address: imarrucho@itqb.unl.pt 
Supporting Information

Table S1. Molar Volumes, $V_{\mathrm{m}, \exp }\left(\mathrm{cm}^{3} \cdot \mathrm{mol}^{-1}\right)$, of the of $\left[\mathrm{C}_{4} \mathrm{mim}\right]\left[\mathrm{NTf}_{2}\right]_{\mathrm{x}}[\mathrm{Y}]_{\mathrm{y}}$ IL mixtures on mole fraction $(x)$ as a function of temperature $(T)$ at pressure $p=0.1 \mathrm{MPa}^{a}$.

\begin{tabular}{|c|c|c|c|c|c|c|c|c|c|c|c|}
\hline \multicolumn{6}{|c|}{$x\left[\mathrm{C}_{4} \mathrm{C}_{1} \mathrm{im}\right]\left[\mathrm{NTf}_{2}\right]+(1-x)\left[\mathrm{C}_{4} \mathrm{C}_{1} \mathrm{im}\right][\mathrm{SCN}]$} & \multicolumn{6}{|c|}{$x\left[\mathrm{C}_{4} \mathrm{C}_{1} \mathrm{im}\right]\left[\mathrm{NTf}_{2}\right]+(1-x)\left[\mathrm{C}_{4} \mathrm{C}_{1} \mathrm{im}\right]\left[\mathrm{N}(\mathrm{CN})_{2}\right]$} \\
\hline \multirow{3}{*}{$T / \mathrm{K}$} & \multicolumn{5}{|c|}{$V_{\mathrm{m}, \exp } /\left(\mathrm{cm}^{3} \cdot \mathrm{mol}^{-1}\right)$} & \multirow{3}{*}{$T / \mathrm{K}$} & \multicolumn{5}{|c|}{$V_{\mathrm{m}, \exp } /\left(\mathrm{cm}^{3} \cdot \mathrm{mol}^{-1}\right)$} \\
\hline & \multicolumn{5}{|c|}{ 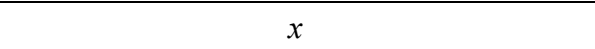 } & & \multicolumn{5}{|c|}{$x$} \\
\hline & 1.000 & 0.748 & 0.500 & 0.250 & 0.000 & & 1.000 & 0.749 & 0.457 & 0.251 & 0.000 \\
\hline 283.15 & 288.86 & 262.65 & 236.22 & 209.60 & 182.82 & 283.15 & 288.86 & 264.76 & 243.84 & 216.69 & 191.83 \\
\hline 288.15 & 289.84 & 263.53 & 236.96 & 210.23 & 183.33 & 288.15 & 289.84 & 265.64 & 244.65 & 217.38 & 192.43 \\
\hline 293.15 & 290.81 & 264.39 & 237.71 & 210.86 & 183.84 & 293.15 & 290.81 & 266.51 & 245.43 & 218.07 & 193.02 \\
\hline 298.15 & 291.80 & 265.26 & 238.45 & 211.49 & 184.36 & 298.15 & 291.80 & 267.41 & 246.21 & 218.76 & 193.60 \\
\hline 303.15 & 292.77 & 266.11 & 239.20 & 212.13 & 184.88 & 303.15 & 292.77 & 268.29 & 247.01 & 219.44 & 194.19 \\
\hline 308.15 & 293.76 & 266.99 & 239.95 & 212.76 & 185.40 & 308.15 & 293.76 & 269.16 & 247.79 & 220.11 & 194.78 \\
\hline 313.15 & 294.73 & 267.85 & 240.70 & 213.40 & 185.90 & 313.15 & 294.73 & 270.05 & 248.58 & 220.81 & 195.37 \\
\hline 318.15 & 295.71 & 268.72 & 241.43 & 214.03 & 186.41 & 318.15 & 295.71 & 270.93 & 249.37 & 221.49 & 195.95 \\
\hline 323.15 & 296.69 & 269.58 & 242.19 & 214.67 & 186.93 & 323.15 & 296.69 & 271.82 & 250.15 & 222.15 & 196.53 \\
\hline 328.15 & 297.68 & 270.46 & 242.94 & 215.29 & 187.44 & 328.15 & 297.68 & 272.71 & 250.94 & 222.84 & 197.12 \\
\hline 333.15 & 298.68 & 271.33 & 243.69 & 215.94 & 187.94 & 333.15 & 298.68 & 273.59 & 251.72 & 223.52 & 197.71 \\
\hline 338.15 & 299.68 & 272.20 & 244.42 & 216.56 & 188.44 & 338.15 & 299.68 & 274.47 & 252.52 & 224.19 & 198.28 \\
\hline 343.15 & 300.67 & 273.08 & 245.18 & 217.20 & 188.97 & 343.15 & 300.67 & 275.36 & 253.30 & 224.88 & 198.88 \\
\hline 348.15 & 301.66 & 273.96 & 245.92 & 217.83 & 189.46 & 348.15 & 301.66 & 276.25 & 254.08 & 225.56 & 199.46 \\
\hline 353.15 & 302.66 & 274.83 & 246.67 & 218.45 & 189.97 & 353.15 & 302.66 & 277.15 & 254.87 & 226.25 & 200.04 \\
\hline 358.15 & 303.65 & 275.71 & 247.42 & 219.08 & 190.48 & 358.15 & 303.65 & 278.04 & 255.66 & 226.93 & 200.61 \\
\hline 363.15 & 304.64 & 276.59 & 248.18 & 219.71 & 190.98 & 363.15 & 304.64 & 278.93 & 256.44 & 227.61 & 201.18 \\
\hline \multicolumn{6}{|c|}{$x\left[\mathrm{C}_{4} \mathrm{C}_{1} \mathrm{im}\right]\left[\mathrm{NTf}_{2}\right]+(1-x)\left[\mathrm{C}_{4} \mathrm{C}_{1} \mathrm{im}\right]\left[\mathrm{C}(\mathrm{CN})_{3}\right]$} & \multicolumn{6}{|c|}{$x\left[\mathrm{C}_{4} \mathrm{C}_{1} \mathrm{im}\right]\left[\mathrm{NTf}_{2}\right]+(1-x)\left[\mathrm{C}_{4} \mathrm{C}_{1} \mathrm{im}\right]\left[\mathrm{BF}_{4}\right]$} \\
\hline \multirow{3}{*}{$T / \mathrm{K}$} & \multicolumn{5}{|c|}{$V_{\mathrm{m}, \exp } /\left(\mathrm{cm}^{3} \cdot \mathrm{mol}^{-1}\right)$} & \multicolumn{6}{|c|}{$V_{\mathrm{m}, \mathrm{exp}} /\left(\mathrm{cm}^{3} \cdot \mathrm{mol}^{-1}\right)$} \\
\hline & \multicolumn{5}{|c|}{$x$} & \multirow{2}{*}{$T / \mathrm{K}$} & \multicolumn{5}{|c|}{$x$} \\
\hline & 1.000 & 0.751 & 0.501 & 0.252 & 0.000 & & 1.000 & 0.751 & 0.498 & 0.250 & 0.000 \\
\hline 283.15 & 288.86 & 271.05 & 253.06 & 234.80 & 216.69 & 283.15 & 288.86 & 263.31 & 238.01 & 212.48 & 186.41 \\
\hline 288.15 & 289.84 & 271.96 & 253.91 & 235.58 & 217.41 & 288.15 & 289.84 & 264.19 & 238.79 & 213.16 & 186.98 \\
\hline 293.15 & 290.81 & 272.88 & 254.77 & 236.36 & 218.13 & 293.15 & 290.81 & 265.06 & 239.55 & 213.82 & 187.54 \\
\hline 298.15 & 291.80 & 273.80 & 255.62 & 237.15 & 218.86 & 298.15 & 291.80 & 265.95 & 240.32 & 214.49 & 188.10 \\
\hline 303.15 & 292.77 & 274.71 & 256.46 & 237.93 & 219.57 & 303.15 & 292.77 & 266.83 & 241.09 & 215.17 & 188.66 \\
\hline 308.15 & 293.76 & 275.63 & 257.32 & 238.71 & 220.29 & 308.15 & 293.76 & 267.70 & 241.86 & 215.83 & 189.22 \\
\hline 313.15 & 294.73 & 276.55 & 258.16 & 239.49 & 220.99 & 313.15 & 294.73 & 268.57 & 242.65 & 216.49 & 189.79 \\
\hline 318.15 & 295.71 & 277.46 & 259.00 & 240.26 & 221.72 & 318.15 & 295.71 & 269.47 & 243.41 & 217.16 & 190.35 \\
\hline 323.15 & 296.69 & 278.37 & 259.85 & 241.05 & 222.43 & 323.15 & 296.69 & 270.35 & 244.21 & 217.83 & 190.90 \\
\hline 328.15 & 297.68 & 279.29 & 260.71 & 241.83 & 223.14 & 328.15 & 297.68 & 271.22 & 244.99 & 218.51 & 191.46 \\
\hline 333.15 & 298.68 & 280.22 & 261.55 & 242.62 & 223.86 & 333.15 & 298.68 & 272.12 & 245.75 & 219.17 & 192.03 \\
\hline 338.15 & 299.68 & 281.13 & 262.42 & 243.39 & 224.59 & 338.15 & 299.68 & 273.00 & 246.54 & 219.84 & 192.59 \\
\hline 343.15 & 300.67 & 282.04 & 263.25 & 244.16 & 225.29 & 343.15 & 300.67 & 273.87 & 247.31 & 220.51 & 193.15 \\
\hline 348.15 & 301.66 & 282.97 & 264.11 & 244.96 & 226.00 & 348.15 & 301.66 & 274.76 & 248.09 & 221.18 & 193.71 \\
\hline 353.15 & 302.66 & 283.90 & 264.95 & 245.72 & 226.72 & 353.15 & 302.66 & 275.66 & 248.88 & 221.85 & 194.28 \\
\hline 358.15 & 303.65 & 284.81 & 265.80 & 246.51 & 227.44 & 358.15 & 303.65 & 276.54 & 249.65 & 222.51 & 194.83 \\
\hline 363.15 & 304.64 & 285.73 & 266.65 & 247.28 & 228.14 & 363.15 & 304.64 & 277.41 & 250.42 & 223.18 & 195.38 \\
\hline
\end{tabular}


Supporting Information

\begin{tabular}{|c|c|c|c|c|c|c|c|c|c|c|c|}
\hline \multicolumn{6}{|c|}{$x\left[\mathrm{C}_{4} \mathrm{C}_{1} \mathrm{im}\right]\left[\mathrm{NTf}_{2}\right]+(1-x)\left[\mathrm{C}_{4} \mathrm{C}_{1} \mathrm{im}\right]\left[\mathrm{PF}_{6}\right]$} & \multicolumn{6}{|c|}{$x\left[\mathrm{C}_{4} \mathrm{C}_{1} \mathrm{im}\right]\left[\mathrm{NTf}_{2}\right]+(1-x)\left[\mathrm{C}_{4} \mathrm{C}_{1} \mathrm{im}\right]\left[\mathrm{CH}_{3} \mathrm{CO}_{2}\right]$} \\
\hline \multirow{3}{*}{$T / \mathrm{K}$} & \multicolumn{5}{|c|}{$V_{\mathrm{m}, \exp } /\left(\mathrm{cm}^{3} \cdot \mathrm{mol}^{-1}\right)$} & \multicolumn{6}{|c|}{$V_{\mathrm{m}, \exp } /\left(\mathrm{cm}^{3} \cdot \mathrm{mol}^{-1}\right)$} \\
\hline & & & $x$ & & & & & & $x$ & & \\
\hline & 1.000 & 0.748 & 0.500 & 0.250 & 0.000 & & 1.000 & 0.749 & 0.498 & 0.250 & 0.000 \\
\hline 283.15 & 288.86 & 268.76 & 248.59 & 228.33 & 208.12 & 283.15 & 288.86 & 263.57 & 238.19 & 212.49 & 186.56 \\
\hline 288.15 & 289.84 & 269.57 & 249.28 & 229.01 & 208.63 & 288.15 & 289.84 & 264.45 & 238.98 & 213.16 & 187.14 \\
\hline 293.15 & 290.81 & 270.46 & 250.09 & 229.75 & 209.31 & 293.15 & 290.81 & 265.32 & 239.76 & 213.85 & 187.75 \\
\hline 298.15 & 291.80 & 271.35 & 250.91 & 230.48 & 209.99 & 298.15 & 291.80 & 266.19 & 240.55 & 214.52 & 188.32 \\
\hline 303.15 & 292.77 & 272.25 & 251.71 & 231.21 & 210.64 & 303.15 & 292.77 & 267.07 & 241.32 & 215.17 & 188.87 \\
\hline 308.15 & 293.76 & 273.15 & 252.52 & 231.93 & 211.29 & 308.15 & 293.76 & 267.95 & 242.07 & 215.83 & 189.43 \\
\hline 313.15 & 294.73 & 274.05 & 253.33 & 232.66 & 211.93 & 313.15 & 294.73 & 268.84 & 242.86 & 216.50 & 190.00 \\
\hline 318.15 & 295.71 & 274.94 & 254.15 & 233.38 & 212.57 & 318.15 & 295.71 & 269.72 & 243.64 & 217.16 & 190.54 \\
\hline 323.15 & 296.69 & 275.85 & 254.96 & 234.10 & 213.20 & 323.15 & 296.69 & 270.62 & 244.41 & 217.83 & 191.09 \\
\hline 328.15 & 297.68 & 276.76 & 255.77 & 234.84 & 213.85 & & 297.68 & 271.51 & & & 191.65 \\
\hline 333.15 & 298.68 & 277.67 & 256.61 & 235.57 & 214.49 & 333.15 & 298.68 & 272.40 & 245.99 & 219.17 & 192.21 \\
\hline 338.15 & 299.68 & 278.57 & 257.43 & 236.30 & 215.13 & 338.15 & 299.68 & 273.28 & 246.78 & 219.86 & 192.77 \\
\hline 343.15 & 300.67 & 279.48 & 258.24 & 237.03 & 215.78 & 343.15 & 300.67 & 274.19 & 247.55 & 220.52 & 193.31 \\
\hline 348.15 & 301.66 & 280.37 & 259.08 & 237.75 & 216.43 & 348.15 & 301.66 & 275.08 & 248.34 & 221.22 & 193.88 \\
\hline 353.15 & 302.66 & 281.29 & 259.89 & 238.50 & 217.07 & & 302.66 & 275.95 & & 221.90 & 194.43 \\
\hline 358.15 & 303.65 & 282.19 & 260.70 & 239.23 & 217.73 & 358.15 & 303.65 & 276.86 & 249.91 & 222.58 & 195.00 \\
\hline 363.15 & 304.64 & 283.10 & 261.53 & 239.94 & 218.35 & 363.15 & 304.64 & 277.74 & 250.70 & 223.26 & 195.54 \\
\hline \multicolumn{6}{|c|}{$x\left[\mathrm{C}_{4} \mathrm{C}_{1} \mathrm{im}\right]\left[\mathrm{NTf}_{2}\right]+(1-x)\left[\mathrm{C}_{4} \mathrm{C}_{1} \mathrm{im}\right]\left[\mathrm{CF}_{3} \mathrm{SO}_{3}\right]$} & \multicolumn{6}{|c|}{$x\left[\mathrm{C}_{4} \mathrm{C}_{1} \mathrm{im}\right]\left[\mathrm{NTf}_{2}\right]+(1-x)\left[\mathrm{C}_{4} \mathrm{C}_{1} \mathrm{im}\right]\left[\left(\mathrm{CH}_{3} \mathrm{O}\right)_{2} \mathrm{PO}_{2}\right]$} \\
\hline \multirow{3}{*}{$T / \mathrm{K}$} & \multicolumn{5}{|c|}{$V_{\mathrm{m}, \exp } /\left(\mathrm{cm}^{3} \cdot \mathrm{mol}^{-1}\right)$} & \multicolumn{6}{|c|}{$V_{\mathrm{m}, \exp } /\left(\mathrm{cm}^{3} \cdot \mathrm{mol}^{-1}\right)$} \\
\hline & \multicolumn{5}{|c|}{$x$} & \multirow{2}{*}{$T / \mathrm{K}$} & \multicolumn{5}{|c|}{$x$} \\
\hline & 1.000 & 0.746 & & 0.250 & 0.000 & & 1.000 & 0.747 & 0.501 & 0.250 & 0.000 \\
\hline 283.15 & 288.86 & 271.91 & 254.74 & 237.35 & 220.34 & & 288.86 & 273.65 & 257.87 & 242.10 & 225.98 \\
\hline 288.15 & 289.84 & 272.81 & 255.58 & 238.12 & 221.03 & 288.15 & 289.84 & 274.56 & 258.75 & 242.88 & 226.66 \\
\hline 293.15 & 290.81 & 273.72 & 256.42 & 238.88 & 221.71 & 293.15 & 290.81 & 275.45 & 259.61 & 243.64 & 227.34 \\
\hline 298.15 & 291.80 & 274.61 & 257.24 & 239.63 & 222.39 & 298.15 & 291.80 & 276.35 & 260.44 & 244.43 & 228.03 \\
\hline 303.15 & 292.77 & 275.51 & 258.08 & 240.40 & 223.08 & 303.15 & 292.77 & 277.26 & 261.28 & 245.20 & 228.74 \\
\hline 308.15 & 293.76 & 276.42 & 258.91 & 241.16 & 223.76 & 308.15 & 293.76 & 278.15 & 262.10 & 245.95 & 229.41 \\
\hline 313.15 & 294.73 & 277.33 & 259.75 & 241.94 & 224.44 & 313.15 & 294.73 & 279.07 & 262.91 & 246.70 & 230.07 \\
\hline 318.15 & 295.71 & 278.25 & 260.59 & 242.71 & 225.12 & & 295.71 & 279.97 & 263.74 & 247.42 & 230.74 \\
\hline 323.15 & 296.69 & 279.15 & 261.42 & 243.47 & 225.81 & 323.15 & 296.69 & 280.88 & 264.56 & 248.17 & 231.41 \\
\hline 328.15 & 297.68 & 280.06 & 262.25 & 244.24 & 226.50 & 328.15 & 297.68 & 281.79 & 265.38 & 248.91 & 232.06 \\
\hline 333.15 & 298.68 & 280.96 & 263.09 & 245.01 & 227.20 & 333.15 & 298.68 & 282.72 & 266.23 & 249.64 & 232.71 \\
\hline 338.15 & 299.68 & 281.88 & 263.93 & 245.80 & 227.88 & 338.15 & 299.68 & 283.62 & 267.06 & 250.37 & 233.37 \\
\hline 343.15 & 300.67 & 282.79 & 264.78 & 246.55 & 228.57 & 343.15 & 300.67 & 284.53 & 267.88 & 251.11 & 234.01 \\
\hline 348.15 & 301.66 & 283.72 & 265.64 & 247.33 & 229.26 & 348.15 & 301.66 & 285.45 & 268.72 & 251.87 & 234.68 \\
\hline 353.15 & 302.66 & 284.64 & 266.48 & 248.11 & 229.95 & 353.15 & 302.66 & 286.38 & 269.54 & 252.60 & 235.32 \\
\hline 358.15 & 303.65 & 285.57 & 267.34 & 248.88 & 230.65 & 358.15 & 303.65 & 287.28 & 270.40 & 253.34 & 235.98 \\
\hline 363.15 & 304.64 & 286.48 & 268.20 & 249.64 & 231.34 & 363.15 & 304.64 & 288.20 & 271.21 & 254.08 & 236.65 \\
\hline
\end{tabular}

${ }^{a}$ Standard uncertainties $u$ are $\mathrm{u}\left(V_{\mathrm{m}}\right)\left(\mathrm{cm}^{-3} \cdot \mathrm{mol}^{-1}\right)$ and $\mathrm{u}(T)=0.02 \mathrm{~K}$, with a expanded uncertainty at the 0.95 confidence level $(k \approx 2)$. 
Supporting Information

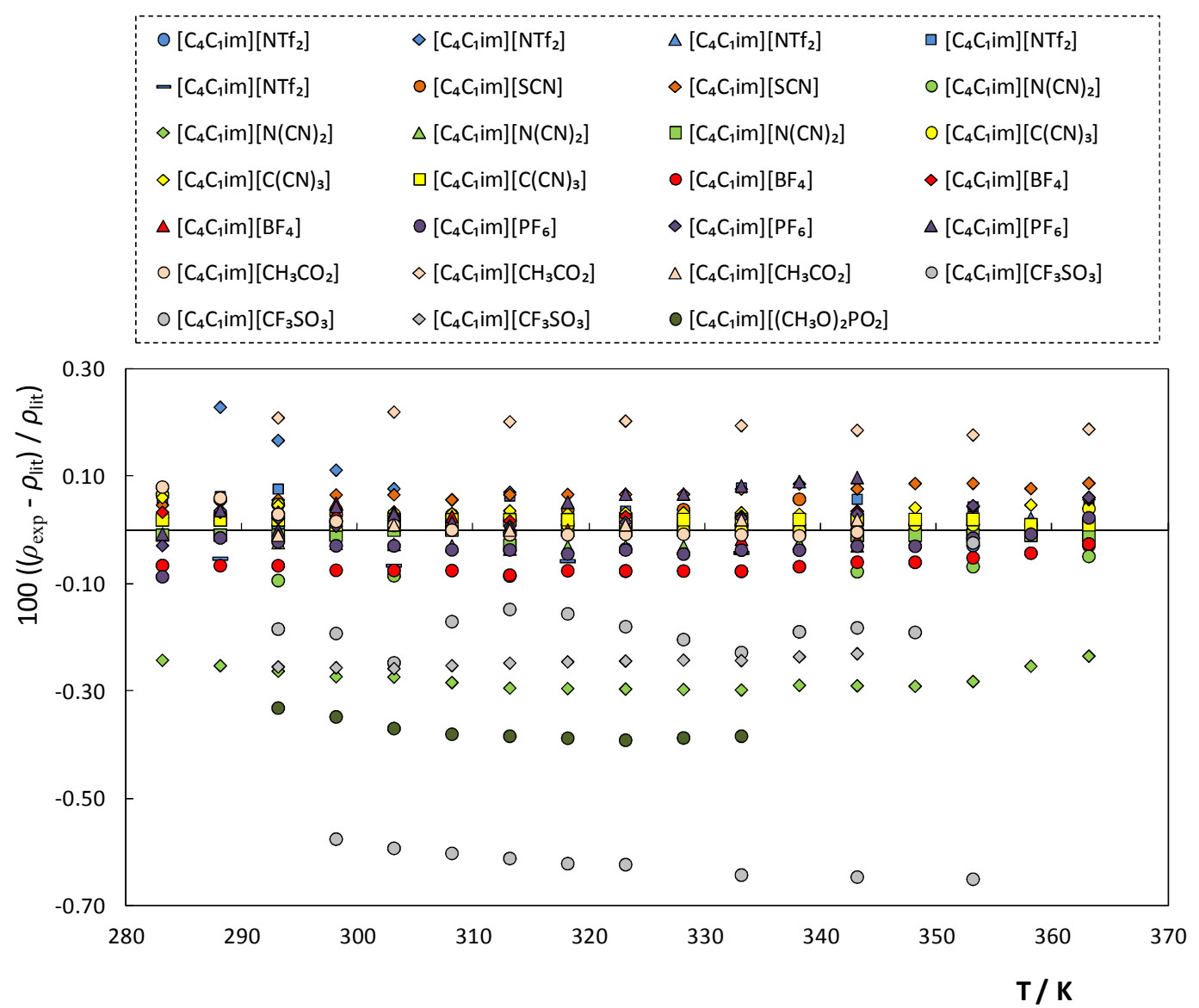

Figure S1. Relative deviations between the experimental $\left(\rho_{\text {exp }}\right)$ and literature $\left(\rho_{\text {lit }}\right)$

density data for pure ILs: different symbols represent different literature sources while the same color corresponds to the same IL. ${ }^{1-18}$ 
Supporting Information
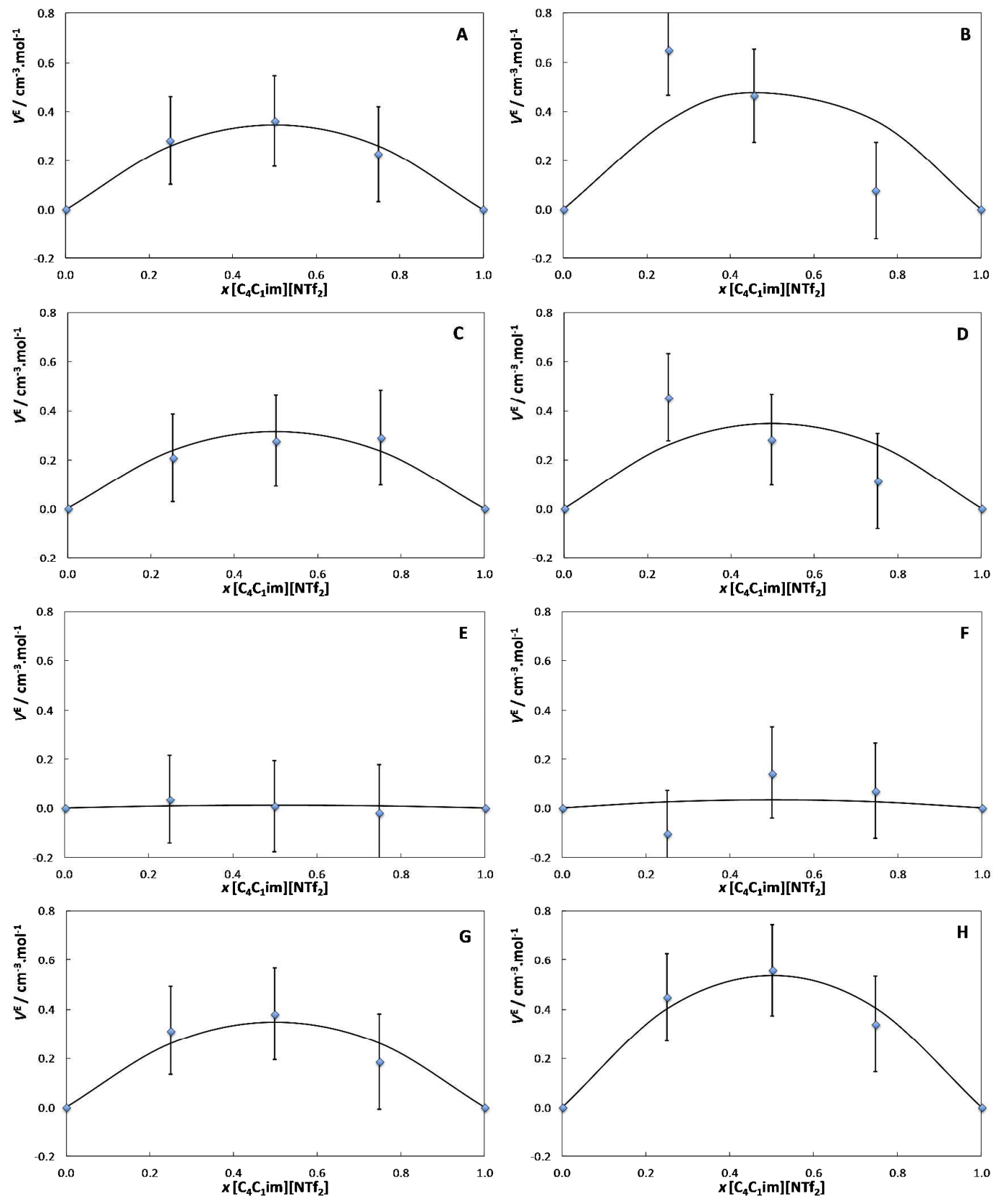

Figure S2. Molar volumes of the studied mixtures: A) $\left[\mathrm{C}_{4} \mathrm{C}_{1} \mathrm{im}\right]\left[\mathrm{NTf}_{2}\right]_{x}[\mathrm{SCN}]_{(1-x)}$, B) $\left[\mathrm{C}_{4} \mathrm{C}_{1} \mathrm{im}\right]\left[\mathrm{NTf}_{2}\right]_{x}\left[\mathrm{~N}(\mathrm{CN})_{2}\right]_{(1-x)}, \quad$ C) $\quad\left[\mathrm{C}_{4} \mathrm{C}_{1} \mathrm{im}\right]\left[\mathrm{NTf}_{2}\right]_{x}\left[\mathrm{C}(\mathrm{CN})_{3}\right]_{(1-x)}, \quad$ D) $\left.\left.\left[\mathrm{C}_{4} \mathrm{C}_{1} \mathrm{im}\right]\left[\mathrm{NTf}_{2}\right]_{x}\left[\mathrm{BF}_{4}\right]_{(1-x)}, \mathbf{E}\right)\left[\mathrm{C}_{4} \mathrm{C}_{1} \mathrm{im}\right]\left[\mathrm{NTf}_{2}\right]_{x}\left[\mathrm{PF}_{6}\right]_{(1-x)}, \mathbf{F}\right)\left[\mathrm{C}_{4} \mathrm{C}_{1} \mathrm{im}\right]\left[\mathrm{NTf}_{2}\right]_{x}\left[\mathrm{CF}_{3} \mathrm{SO}_{3}\right]_{(1-}$ $x$, G) $\left[\mathrm{C}_{4} \mathrm{C}_{1} \mathrm{im}\right]\left[\mathrm{NTf}_{2}\right]_{x}\left[\mathrm{CH}_{3} \mathrm{CO}_{2}\right]_{(1-x)}$, and $\left.\mathbf{H}\right)\left[\mathrm{C}_{4} \mathrm{C}_{1} \mathrm{im}\right]\left[\mathrm{NTf}_{2}\right]_{x}\left[\left(\mathrm{CH}_{3} \mathrm{O}\right)_{2} \mathrm{PO}_{2}\right]_{(1-x)}$, at 298.15 K and 0.1 MPa. Blue diamond represents experimental molar volume; straight lines represent the ideal behavior described by Equation 3. 
Supporting Information

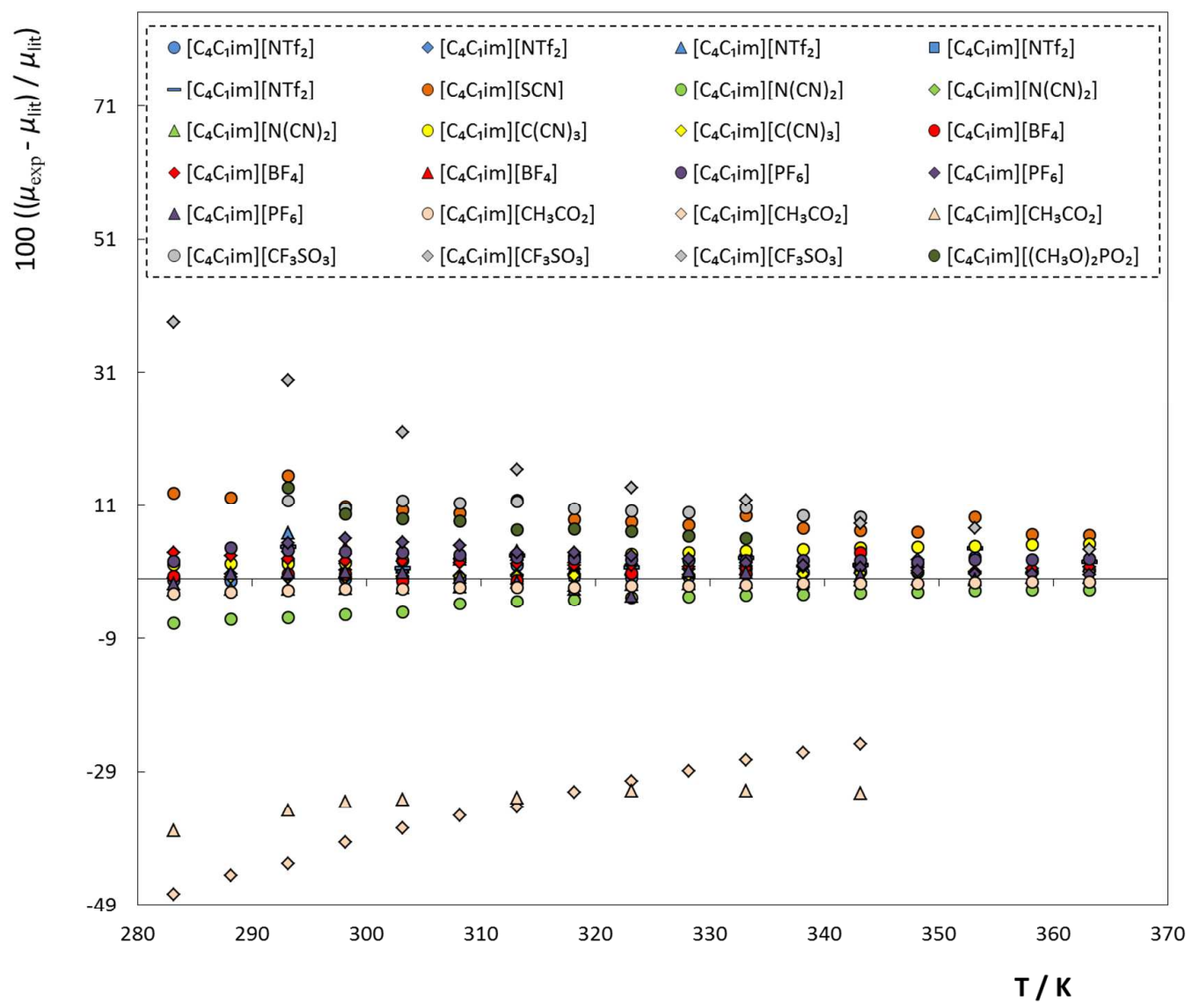

Figure S3. Relative deviations between the experimental $\left(\eta_{\text {exp }}\right)$ and literature $\left(\eta_{l i t}\right)$ viscosity data: different symbols represent different literature sources while the same color corresponds to the same IL. . $^{3,4,7,8,10,13,14,17-26}$ 


\section{Supporting Information}

\section{References}

(1) Zhang, Y.; Zhang, T.; Gan, P.; Li, H.; Zhang, M.; Jin, K.; Tang, S. Solubility of Isobutane in Ionic Liquids [BMIm][PF6], [BMIm] [BF4], and [BMIm] [Tf2N]. J. Chem. Eng. Data 2015, 60, 1706-1714.

(2) Jacquemin, J.; Husson, P.; Majer, V.; Costa Gomes, M. F. Influence of the Cation on the Solubility of $\mathrm{CO} 2$ and $\mathrm{H} 2$ in Ionic Liquids Based on the Bis(trifluoromethylsulfonyl)imide Anion. J. Solution Chem. 2007, 36, 967-979.

(3) Harris, K. R.; Kanakubo, M.; Woolf, L. A. Temperature and Pressure Dependence of the Viscosity of the Ionic Liquids 1-Hexyl-3-methylimidazolium Hexafluorophosphate and 1-Butyl-3-methylimidazolium Bis(trifluoromethylsulfonyl)imide. J. Chem. Eng. Data 2007, 52, 1080-1085.

(4) Salgado, J.; Regueira, T.; Lugo, L.; Vijande, J.; Fernández, J.; García, J. Density and viscosity of three (2,2,2-trifluoroethanol + 1-butyl-3-methylimidazolium) ionic liquid binary systems. J. Chem. Thermodyn. 2014, 70, 101-110.

(5) Nieto de Castro, C. A.; Langa, E.; Morais, A. L.; Lopes, M. L. M.; Lourenço, M. J. V.; Santos, F. J. V.; Santos, M. S. C. S.; Lopes, J. N. C.; Veiga, H. I. M.; Macatrão, M.; Esperança, J. M. S. S.; Marques, C. S.; Rebelo, L. P. N.; Afonso, C. A. M. Studies on the density, heat capacity, surface tension and infinite dilution diffusion with the ionic liquids [C4mim][NTf2], [C4mim][dca], [C2mim][EtOSO3] and [Aliquat][dca]. Fluid Phase Equilib. 2010, 294, 157-179.

(6) Domańska, U.; Królikowska, M. Effect of temperature and composition on the surface tension and thermodynamic properties of binary mixtures of 1-butyl-3methylimidazolium thiocyanate with alcohols. J. Colloid Interface Sci. 2010, 348, 661667.

(7) Carvalho, P. J.; Regueira, T.; Santos, L.; Fernandez, J.; Coutinho, J. A. P. Effect of Water on the Viscosities and Densities of 1-Butyl-3-methylimidazolium Dicyanamide and 1-Butyl-3-methylimidazolium Tricyanomethane at Atmospheric Pressure. J. Chem. Eng. Data 2010, 55, 645-652.

(8) Seoane, R. G.; Corderí, S.; Gómez, E.; Calvar, N.; González, E. J.; Macedo, E. A.; Domínguez, Á. Temperature Dependence and Structural Influence on the Thermophysical Properties of Eleven Commercial Ionic Liquids. Ind. Eng. Chem. Res. 2012, 51, 2492-2504. 
Supporting Information

(9) Koller, T. M.; Schmid, S. R.; Sachnov, S. J.; Rausch, M. H.; Wasserscheid, P.; Fröba, A. P. Measurement and Prediction of the Thermal Conductivity of Tricyanomethanide- and Tetracyanoborate-Based Imidazolium Ionic Liquids. Int. J. Thermophys. 2014, 35, 195-217.

(10) Harris, K. R.; Kanakubo, M.; Woolf, L. A. Temperature and Pressure Dependence of the Viscosity of the Ionic Liquid 1-Butyl-3-methylimidazolium Tetrafluoroborate: Viscosity and Density Relationships in Ionic Liquids. J. Chem. Eng. Data 2007, 52, 2425-2430.

(11) Soriano, A. N.; Doma Jr, B. T.; Li, M.-H. Measurements of the density and refractive index for 1-n-butyl-3-methylimidazolium-based ionic liquids. J. Chem. Thermodyn. 2009, 41, 301-307.

(12) Pereiro, A. B.; Legido, J. L.; Rodri'guez, A. Physical properties of ionic liquids based on 1-alkyl-3-methylimidazolium cation and hexafluorophosphate as anion and temperature dependence. J. Chem. Thermodyn. 2007, 39, 1168-1175.

(13) Hiraga, Y.; Kato, A.; Sato, Y.; Smith, R. L. Densities at Pressures up to 200 $\mathrm{MPa}$ and Atmospheric Pressure Viscosities of Ionic Liquids 1-Ethyl-3methylimidazolium Methylphosphate, 1-Ethyl-3-methylimidazolium Diethylphosphate, 1-Butyl-3-methylimidazolium Acetate, and 1-Butyl-3-methylimidazolium Bis(trifluoromethylsulfonyl)imide. J. Chem. Eng. Data 2015, 60, 876-885.

(14) Araújo, J. M. M.; Pereiro, A. B.; Alves, F.; Marrucho, I. M.; Rebelo, L. P. N. Nucleic acid bases in 1-alkyl-3-methylimidazolium acetate ionic liquids: A thermophysical and ionic conductivity analysis. J. Chem. Thermodyn. 2013, 57, 1-8.

(15) Haghtalab, A.; Shojaeian, A. Volumetric and viscometric behaviour of the binary systems of N-methyldiethanolamine and diethanolamine with 1-butyl-3methylimidazolium acetate at various temperatures. J. Chem. Thermodyn. 2014, 68, 128-137.

(16) Klomfar, J.; Součková, M.; Pátek, J. Temperature Dependence Measurements of the Density at 0.1 MPa for 1-Alkyl-3-methylimidazolium-Based Ionic Liquids with the Trifluoromethanesulfonate and Tetrafluoroborate Anion. J. Chem. Eng. Data 2010, 55, 4054-4057.

(17) Gong, Y.-h.; Shen, C.; Lu, Y.-z.; Meng, H.; Li, C.-x. Viscosity and Density Measurements for Six Binary Mixtures of Water (Methanol or Ethanol) with an Ionic 
Liquid ([BMIM][DMP] or [EMIM][DMP]) at Atmospheric Pressure in the Temperature Range of (293.15 to 333.15) K. J. Chem. Eng. Data 2012, 57, 33-39.

(18) Neves, C. M. S. S.; Kurnia, K. A.; Coutinho, J. A. P.; Marrucho, I. M.; Lopes, J. N. C.; Freire, M. G.; Rebelo, L. P. N. Systematic study of the thermophysical properties of imidazolium-based ionic liquids with cyano-functionalized anions. J. Phys. Chem. B 2013, 117, 10271-10283.

(19) Vraneš, M.; Zec, N.; Tot, A.; Papović, S.; Dožić, S.; Gadžurić, S. Density, electrical conductivity, viscosity and excess properties of 1-butyl-3-methylimidazolium bis(trifluoromethylsulfonyl)imide + propylene carbonate binary mixtures. J. Chem. Thermodyn. 2014, 68, 98-108.

(20) Salinas, R.; Pla-Franco, J.; Lladosa, E.; Montón, J. B. Density, Speed of Sound, Viscosity, and Excess Properties of Binary Mixtures Formed by Ethanol and Bis(trifluorosulfonyl)imide-Based Ionic Liquids. J. Chem. Eng. Data 2015, 60, 525540.

(21) Sanmamed, Y. A.; González-Salgado, D.; Troncoso, J.; Cerdeiriña, C. A.; Romaní, L. Viscosity-induced errors in the density determination of room temperature ionic liquids using vibrating tube densitometry. Fluid Phase Equilib. 2007, 252, 96-102.

(22) Rocha, M. A. A.; Ribeiro, F. M. S.; Ferreira, A. I. M. C. L.; Coutinho, J. A. P.; Santos, L. M. N. B. F. Thermophysical properties of [CN - 1C1im][PF6] ionic liquids. J. Mol. Liq. 2013, 188, 196-202.

(23) Fan, W.; Zhou, Q.; Sun, J.; Zhang, S. Density, Excess Molar Volume, and Viscosity for the Methyl Methacrylate + 1-Butyl-3-methylimidazolium Hexafluorophosphate Ionic Liquid Binary System at Atmospheric Pressure. J. Chem. Eng. Data 2009, 54, 2307-2311.

(24) Crosthwaite, J. M.; Muldoon, M. J.; Dixon, J. K.; Anderson, J. L.; Brennecke, J. F. Phase transition and decomposition temperatures, heat capacities and viscosities of pyridinium ionic liquids. J. Chem. Thermodyn. 2005, 37, 559-568.

(25) Almeida, H. F. D.; Passos, H.; Lopes-da-Silva, J. A.; Fernandes, M. A.; Freire, M. G.; Coutinho, J. A. P. Thermophysical Properties of Acetate-Based Ionic Liquids. J. Chem. Eng. Data 2012, 57, 3005-3013.

(26) Kenneth, R. S.; Annegret, S.; María-José, T.: Viscosity and Density of 1-Alkyl3-methylimidazolium Ionic Liquids. In Clean Solvents; American Chemical Society, 2002; Vol. 819; pp 34-49. 